\title{
The Rothman Index: Assessing risk for 30-day readmission in gynecologic oncology service patients
}

\author{
Anh T. Butz, Michel El Alam, William P. Irvin Jr., Dale W. Stovall \\ Riverside Regional Medical Center Department of Obstetrics and Gynecology, Newport News, VA, United States
}

Received: February 6, 2019

Accepted: March 27, 2019

Online Published: April 10, 2019

DOI: $10.5430 /$ jha.v8n2p54

URL: https://doi.org/10.5430/jha.v8n2p54

\begin{abstract}
Background: The Rothman Index (RI) is a previously validated, continuously computed score derived from 26 clinical measures that assesses a patient's clinical status. It has been used as a prognostic indicator in the intensive care setting and has been incorporated into the electronic medical record. This study was designed to determine its utility in assessing 30-day readmission rates in postoperative gynecologic oncology service patients.

Methods: In this retrospective case-control study, gynecologic oncology service surgical patients readmitted within 30 days of discharge (cases) were matched 1:2 by procedure, diagnosis, age and comorbidities to non-readmitted gynecologic oncology surgical patients (controls). All procedures were performed at one center by a single surgeon. Rothman Index scores were obtained immediately postoperative $\left(\mathrm{RI}_{p}\right)$ and before discharge $\left(\mathrm{RI}_{d}\right)$, and the difference between these values was calculated $\left(\mathrm{RI}_{c}\right)$. Scores were compared between cases and controls.

Results: In total, 24 cases were matched to 48 controls. The mean age of all study participants was 56 years. The $\mathrm{RI}_{d}$ was significantly different between groups, with median $\mathrm{RI}_{d}$ of 70 for readmitted subjects and 75.5 for controls $(P=.029)$. Binomial regression of readmission on $\mathrm{RI}$ revealed an $\mathrm{RI}_{d}$ of 58.9 or less was associated with at least $50 \%$ likelihood of readmission $(P=$ .017). Cases and controls did not significantly differ based on $\mathrm{RI}_{p}$ or $\mathrm{RI}_{c}$, and were similar with respect to risk factors including diabetes, smoking, and BMI.

Conclusion: Delaying discharge for patients with RI below a designated threshold may reduce gynecologic oncology postoperative readmission rates.
\end{abstract}

Key Words: Rothman Index, Postoperative, Readmission, Gynecologic oncology

\section{INTRODUCTION}

In-patient assessment and management of postoperative gynecologic oncology patients can be challenging. These patients tend to have multiple comorbidities in addition to their cancer diagnosis, and may have undergone an extensive surgical intervention. The timing for hospital discharge of these patients may also be challenging. To determine the appropriate day for discharge, clinicians must assimilate information from many sources. Included are their physical examination, history, vital signs, results from imaging, laboratory tests, nursing assessments, and information from other health care providers involved in the patient's care. For a variety of reasons, these patients are frequently readmitted to the hospital within a few weeks from the day of discharge. A literature search of 30-day readmissions in the gynecologic oncology population revealed rates from $4.5 \%$ to $13.2 \% .^{[1-4]}$ Among patients undergoing index surgery for stage IIIc to IV ovarian cancer, nearly $20 \%$ are readmitted within 30 days. ${ }^{[5]}$ One States. 
potential cause of early readmissions is the discharging of patients before they are clinically ready.

High readmission rates that occur within a relatively short interval of time post-discharge are not acceptable. Health care centers that have an excessively high rate of readmission over a 30-day post-discharge interval also suffer financial consequences for their readmission rates. ${ }^{[6]}$ Access to a tool that could assist clinicians in determining an appropriate date for discharge could reduce early readmission rates.

The Rothman Index (RI), a composite score from 0 to 100 , is a previously validated measure of a patient's overall clinical well-being. ${ }^{[7]}$ This patient assessment tool has been integrated into the electronic medical record (EMR). It is a single score that is computed from 26 clinical variables that are frequently updated within the EMR (see Table 1). The RI was developed in an effort to track a patient's well-being or deterioration over time. Clinical variables included in the RI are vital signs, laboratory values, and nursing assessments. A patient's RI score is re-calculated and reported frequently throughout the day as new data are obtained, and it is plotted graphically. Therefore, up-to-date trends and changes in a patient's RI can be reviewed by the treating clinician. The RI has been previously studied as a prognostic indicator in the surgical intensive care unit (SICU) setting ${ }^{[8]}$ and in both general medicine and surgery patients. ${ }^{[9]}$ In some cases, the RI has been shown to outperform similar indices such as the Modified Early Warning Score ${ }^{[10]}$ (MEWS) and vital signs-based predictors. ${ }^{[11]}$ Specifically, a retrospective study of over 32,000 patient visits found the RI had greater sensitivity and specificity than the MEWS in predicting 24-hour mortality.

Table 1. Rothman Index input variables

\begin{tabular}{llllll}
\hline \multicolumn{2}{l}{ Vital signs } & & Nursing Assessments & & Tests (blood work and telemetry) \\
\hline 1. & Temperature & 7. & Cardiac & 19. & Creatinine \\
2. & Diastolic blood pressure & 8. & Respiratory & 20. & Sodium \\
3. & Systolic blood pressure & 9. & Gastrointestinal & 21. & Chloride \\
4. & Pulse oximetry & 10. & Genitourinary & 22. & Potassium \\
5. & Respiration rate & 11. & Neurological & 23. & Blood urea nitrogen \\
6. & Heart rate & 12. & Skin & 24. & White blood cell \\
& & 13. & Safety & 25. & Hemoglobin \\
& 14. & Peripheral vascular & 26. & Cardiac rhythm (one of the following values: \\
& 15. & Food/nutrition & & asystole, sinus rhythm, sinus bradycardia, sinus \\
& 16. & Psychosocial & & tachycardia, atrial fibrillation, atrial flutter, heart \\
& 17. & Musculoskeletal & & block, junctional rhythm, paced, ventricular \\
& 18. & Braden score & & fibrillation, ventricular tachycardia) \\
\hline
\end{tabular}

Note. Values of the variables listed are used to compute a Rothman Index score that ranges from 0 to 100 and reflects a patient's overall well-being. In our system, a new score is computed whenever new values are entered using the most recent values of all variables. Adapted from "Development and validation of a continuous measure of patient condition using the Electronic Medical Record,” MJ Rothman, SI Rothman, J Beals, 2013, Journal of Biomedical Informatics, 46(5):837-848.

To date, no published studies have examined the application of the RI in either the benign gynecology patient population or in the gynecologic oncology setting. As an automated, quantitative measure of patient status, the RI has potential as a decision-making tool that is easy to use and reliable. The objective of this pilot study was to examine the utility of the RI in assessing 30-day readmission risks in a postoperative gynecologic oncology patient service in women undergoing their initial gynecologic oncology procedure. We hypothesized that either a change over time or an absolute threshold of the RI could be successfully used to distinguish between patients who underwent readmission within 30 days and those who did not.

\section{MATERIALS AND METHODS}

This matched, case-control study was conducted as part of our hospital system's Sepsis Early Detection Initiative, which

Published by Sciedu Press was approved by the Riverside Health System Institutional Review Board. All patients included in the study underwent surgery by a single gynecologic oncology surgeon at one medical center between January 2014 and March 2016. Patients readmitted within 30 days of discharge (cases) were matched $1: 2$ by admitting diagnosis, primary surgical procedure, and age to non-readmitted patients (controls). Matching criteria were obtained from the operative report. Diagnoses included cervical cancer, endometriosis, leiomyoma, menorrhagia, ovarian neoplasm, uterine cancer, and vulvar neoplasm. Procedures included laparotomy, hysterectomy, adnexal surgery (salpingectomy and/or oophorectomy), adhesiolysis, lymphadenectomy, vulvectomy, and bowel/omental resection. Outpatient surgeries and admissions terminated by patient expiration were not included in the initial subject pool query. Subjects were excluded if they had prior gynecologic oncology treatment - including surgery, radiation, 
or chemotherapy — or if the subject passed away within 30 days of the procedure. Observation admissions for less than 24 hours were not considered readmissions. When multiple potential controls were available, subjects were also matched, in order, for presence of diabetes mellitus, presence of smoking history, and basal metabolic index (BMI); this information was obtained from the admission note or preoperative survey on the day of surgery. Diabetes status was defined as diagnosis of diabetes at time of surgery. Smoking status was defined as history of current or former smoking. Of note, although the RI was integrated and available as part of the EMR during the interval of the study, it was neither accessed by the gynecologic oncology service, nor was it utilized for clinical decision-making during the study interval. For subject recruitment overview, see Figure 1.

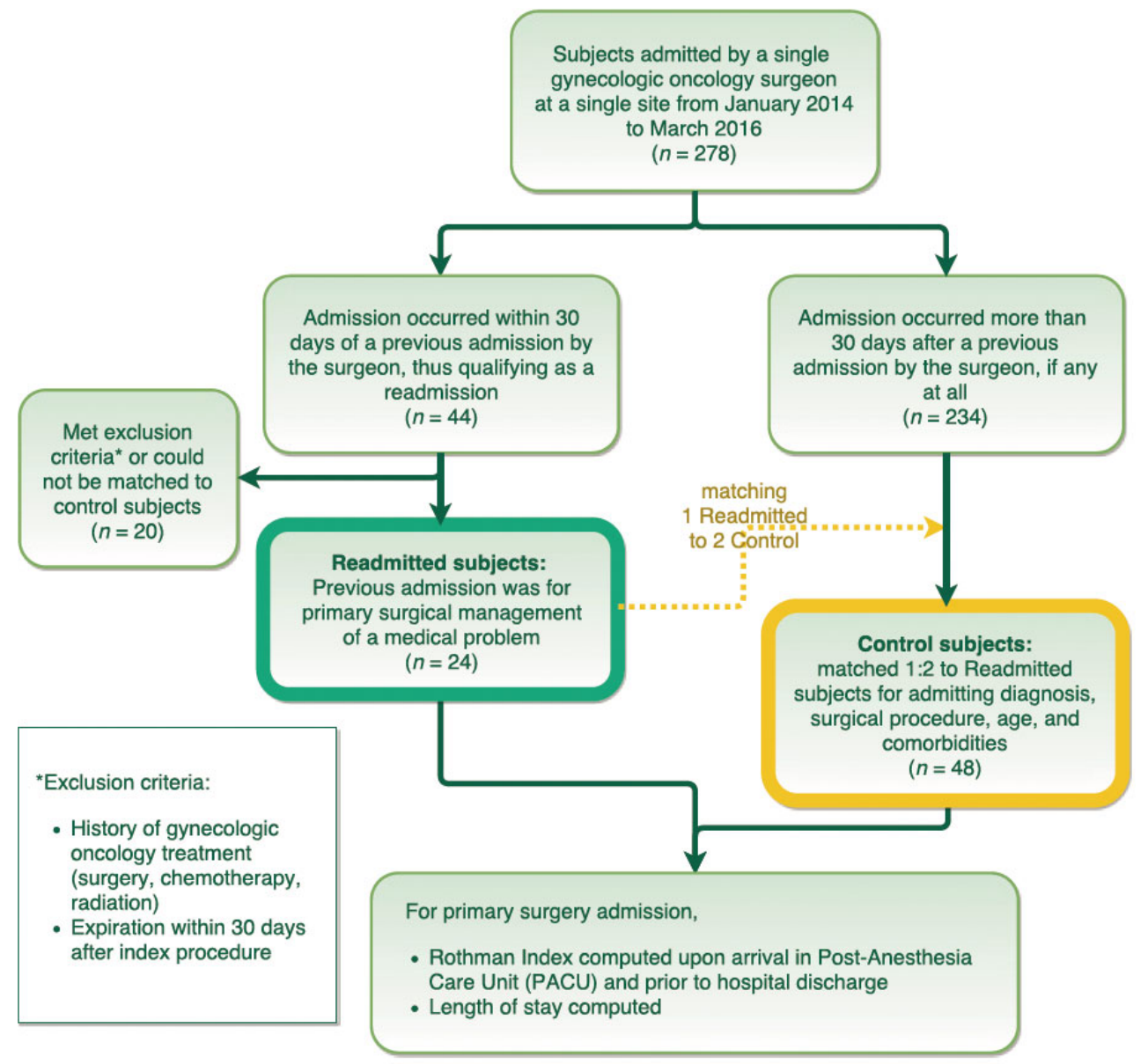

Figure 1. Subject recruitment

Our power analysis was based on a preliminary survey of the RI on the day of discharge for both readmitted and control subjects who had been treated in our hospital. To calculate the sample size needed to achieve at least $80 \%$ statistical power $(\beta=0.2)$ to detect a 10 -point difference in the RI at discharge with a two-sided significance level of $\alpha=0.05$, we determined that at least 21 cases were needed while maintaining a ratio of 2 controls per case subject.

For each subject, RI values obtained immediately postoperative $\left(\mathrm{RI}_{p}\right)$ and just prior to discharge $\left(\mathrm{RI}_{d}\right)$ were abstracted from the EMR, and the difference between these values was calculated $\left(\mathrm{RI}_{c}\right)$. Readmission was analyzed with respect to $\mathrm{RI}_{p}, \mathrm{RI}_{d}$, and $\mathrm{RI}_{c}$ using Wilcoxon rank sum test and binomial regression methods where indicated. Differences in baseline demographic information were analyzed using Wilcoxon (age, BMI) and Fisher (race, diabetes, smoking) tests. Length of stay was also analyzed as an alternate predictor of readmission. All statistics were computed using $\mathrm{R}$ software.

\section{RESULTS}

In total, 24 cases and 48 controls were included in the study. Readmission and control subjects were similar with respect to age, race, BMI, diabetes status, and smoking status (see Table 2).

ISSN 1927-6990 E-ISSN 1927-7008 
Table 2. Subject demographics

\begin{tabular}{llll}
\hline Demographic parameter & Readmitted $(\boldsymbol{n}=\mathbf{2 4})$ & Control $(\boldsymbol{n}=\mathbf{4 8})$ & $\boldsymbol{P}$ \\
\hline Age in years $(95 \%$ CI) & $55.0(49.4-60.6)$ & $56.7(52.7-60.8)$ & .62 \\
Race distribution of Asian, Black, & $0(0 \%), 10(42 \%), 1(4 \%), 13(54 \%)$ & $2(4 \%), 14(29 \%), 1(2 \%), 31(65 \%)$ & .49 \\
Hispanic, non-Hispanic White & $37.2(31.4-43.1)$ & $34.8(31.7-37.9)$ & .50 \\
BMI in $\mathrm{kg} / \mathrm{m}^{2}$ (95\% CI) & $6(25.0 \%)$ & $10(20.8 \%)$ & .77 \\
Subjects with diabetes & $16(33.3 \%)$ & .60 \\
\hline Subjects with history of smoking & $10(41.7 \%)$ & \\
\hline
\end{tabular}

Note. Data reflect status on admission for primary procedure and were not significantly different between readmitted and control subjects. Statistics computed using Wilcoxon (age, BMI) and Fisher (race, diabetes, smoking) tests.

The RI at time of discharge $\left(\mathrm{RI}_{d}\right)$ was significantly different between readmitted subjects and controls $(P=.029$, see Figure 2(a)). Median $\mathrm{RI}_{d}$ was 70 for readmitted subjects and 75.5 for controls. The RI in the immediate postoperative period $\left(\mathrm{RI}_{p}\right)$ did not significantly differ between groups;
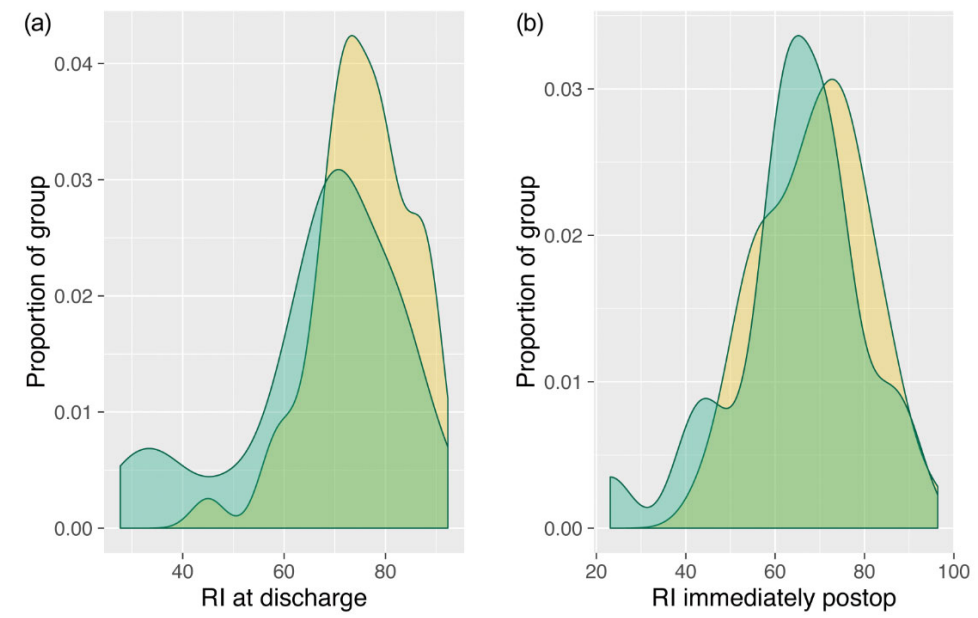

Figure 2. Rothman Index during primary gynecologic surgical admission Kernel density estimates of $R I_{d}(2(a)), R I_{p}(2(b))$, and $R I_{c}(2(c))$. Readmitted subjects significantly differed from controls with respect to $R I_{d}$, but not $R I_{p}$ or $R I_{c}$.
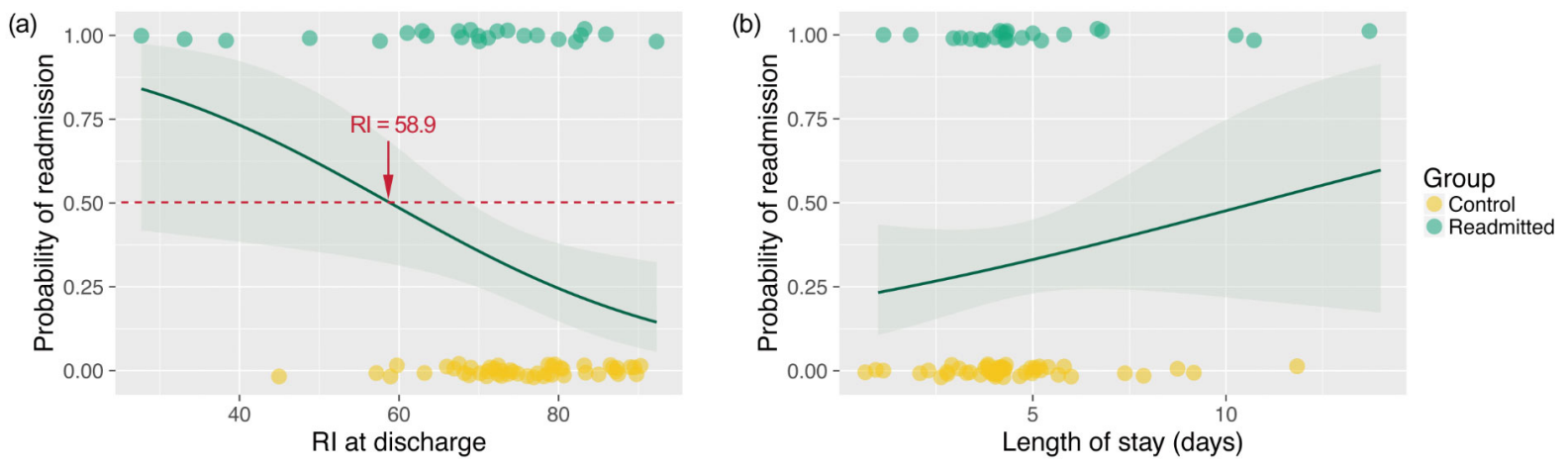

Figure 3. Predictors of readmission after initial gynecologic surgery

Binomial regression models of readmission as predicted by $R I_{d}(a)$ and length of stay $(b)$. Dots represent data points used to generate model, solid line represents the model itself, and shading represents 95\% confidence interval. In (a), dotted line demarcates 50\% probability of readmission; its intersection with the model is highlighted. 
Based on the finding of a significant difference in $\mathrm{RI}_{d}$ between readmitted subjects and controls, binomial regression was used to identify the relationship between $\mathrm{RI}_{d}$ and likelihood of readmission. The regression model was statistically significant $(P=.017)$ and predicted that $\mathrm{RI}_{d}$ of 58.9 or less is associated with at least $50 \%$ chance of readmission (see Figure 3(a)). For comparison, length of stay of the primary surgical admission was empirically chosen as another potential predictor of readmission; this model was not significant (see Figure 3(b)). Of note, one outlier was excluded in the model based on length of stay due to admission lasting 33 days.

Readmission latency, defined as the number of days to readmission, was examined with respect to $\mathrm{RI}_{d}$ and $\mathrm{RI}_{c}$. The majority of readmissions occurred within a week of discharge. $\mathrm{RI}_{d}$ weakly correlated with the number of days to readmission, approaching significance ( $P=.07$, Pearson's $r=-0.38$ ). The interval change $\mathrm{RI}_{c}$ was not predictive of readmission latency.

To account for the effect of disease severity on readmission, the cohorts were evaluated for differences in cancer staging. Staging was determined according to standards set forth by the International Federation of Gynecology and Obstetrics (FIGO). The readmitted and control subjects were similarly distributed across cancer staging categories (see Table 3). A distinction was made between benign pathology and precancerous lesions, which included endometrial hyperplasia and cervical intraepithelial neoplasia. While providing more description of the data, these staging categories decrease statistical power to detect a difference between readmitted and control subjects. Therefore, the staging was simplified into a "low" cancer burden group - which combines subjects with benign, precancerous, or stage I disease - and a "high" cancer burden group - which combines the remaining subjects with stages II, III, and IV disease. As such, the readmission cohort had 9 subjects with "high" cancer burden and 15 subjects with "low" cancer burden, while the control cohort had 11 subjects with "high" cancer burden and 37 subjects with "low" cancer burden. Analysis of this simplified categorization of disease severity did not find any group differences.

Table 3. Stage of cancer in index surgical admission

\begin{tabular}{lllllll}
\hline & \multicolumn{7}{c}{ Cancer Staging } \\
\cline { 2 - 7 } & Benign & Precancerous & I & II & III & IV \\
\hline Readmitted & $8(33 \%)$ & $3(13 \%)$ & $4(17 \%)$ & $2(8.3 \%)$ & $6(25 \%)$ & $1(4.2 \%)$ \\
Control & $14(29 \%)$ & $7(15 \%)$ & $16(33 \%)$ & $4(8.3 \%)$ & $5(10 \%)$ & $2(4.2 \%)$ \\
\hline
\end{tabular}

Note. Count (percentage of cohort) of subjects staged according to FIGO standards based on clinical findings and surgical pathology. Pattern of cancer staging was not significantly different between readmitted subjects and control (Fisher's exact, $P=.26$ ); see text for detail on analysis.

Upon discharge from the index admission, subjects were discharged to home with self-care, home with home health service, skilled nursing facility (SNF), or long-term acute care facility (LTAC). The pattern of discharge disposition was not significantly different between cases and controls (see Table 4). For readmitted subjects, the readmission diagnoses varied, including postoperative bleeding $(n=3)$, infection ( $\mathrm{n}$
$=10)$, and gastrointestinal (GI) complication $(\mathrm{n}=6)$, as well as rarer events such as seizure, renal failure, respiratory failure, and pulmonary embolism ( $\mathrm{n}=1$ for each). In a general linear model, readmission diagnoses of bleeding or infection were associated with decreased RI on discharge from the index admission (see Figure 4, $P<.01$ ). Subjects readmitted for GI complication had $\mathrm{RI}_{d}$ comparable to controls.

Table 4. Patient disposition from index surgical admission

\begin{tabular}{lllll}
\hline & \multicolumn{3}{l}{ Disposition } & \\
\cline { 2 - 5 } & Home & Home with Home Health & Skilled Nursing Facility & Long-Term Acute Care Facility \\
\hline Readmitted & $16(67 \%)$ & $4(17 \%)$ & $3(13 \%)$ & $1(4.2 \%)$ \\
Control & $36(75 \%)$ & $9(19 \%)$ & $3(6.2 \%)$ & $0(0 \%)$ \\
\hline
\end{tabular}

Note. Count (percentage of cohort) of subjects discharged to four different settings. Discharge disposition pattern was not significantly different between readmitted subjects and control (Fisher's exact, $P=.43$ ). 


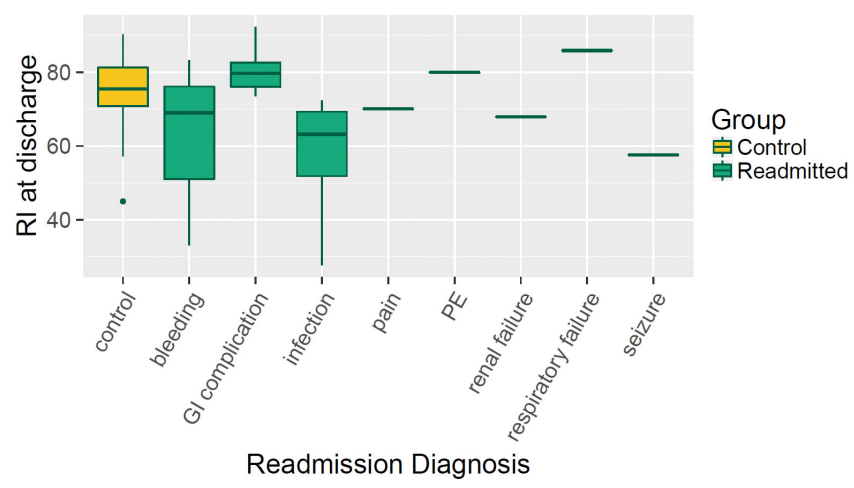

Figure 4. Readmission diagnoses and associated Rothman Index at discharge from primary admission

Boxplots of RI values at discharge $\left(R I_{d}\right)$ from the primary surgical admission, grouped by readmission diagnosis, with non-readmitted control group displayed for reference. Box represents median and interquartile range (IQR, 25th to 75 th percentiles) with whiskers extending to minimum and maximum values, excluding outliers (defined as outside the IQR by more than 1.5 times the IQR,

denoted as dots). In a general linear model of the 24 subjects who required readmission within 30 days of the index admission, readmission diagnoses of bleeding or infection were associated with lower values of $R I_{d}$.

\section{Discussion}

Gynecologic oncology service patients commonly have multiple comorbidities, undergo invasive procedures, and because of the demographics of gynecologic cancer, tend to be somewhat older than their benign gynecologic surgery counterparts. Therefore, their complexity of care, length of stay, and morbidity and mortality are increased as compared to a benign gynecologic service. All of these variables may result in an increase in both early and late readmission rates in this population.

During these patients' hospital stay, numerous data points are obtained including historical information, physical assessments, laboratory values, and imaging. To make the best clinical decision for each patient, the clinician must take all of these data into account. Therefore, identification of a tool that can assist healthcare providers in medical decision-making, especially in a complex patient care setting, is important.

We found that a tool in which many clinical variables are synthesized into a single score, the RI, can assist clinicians in identifying gynecologic oncology patients who are not yet ready for discharge from the hospital as they are at increased risk for early readmission. In this regard, the data point that was most useful was the value of the RI on the day of discharge. More specifically, a threshold of 58.9 was associated with a $50 \%$ chance for readmission. This finding

Published by Sciedu Press is consistent with the RI's empirically derived thresholds, in which a RI of 65 represents the typical acuity of patients discharged to a skilled nursing or rehabilitation facility, and a RI of 40 or less indicates possible critical status and the need for a higher level of care. ${ }^{\text {[7] }}$

The value of the RI in the immediate postoperative period was not predictive of outcome, nor was the interval difference in the RI between the immediate postoperative period and the day of discharge. These results do not imply that neither absolute values of the RI nor its trends are uninformative when making medical decisions, including when a change in therapy is indicated. It does demonstrate, however, that even when an experienced clinician thinks that it is appropriate to discharge a patient, a tool like the RI can help distinguish between those individuals who are and are not ready for discharge.

Patients who made up the cases and controls in this study were similar in many respects. Specifically, their overall medical condition at the time of entry into the study, based on $\mathrm{RI}_{p}$, was not significantly different. Furthermore, the majority of both groups did have an interval difference in $\mathrm{RI}_{c}$ of 0 or greater, which indicates that both cases and controls maintained their medical status or improved. In contrast to our results, previous analysis of patients treated in a SICU setting which assessed 48-hour readmission rates found that readmitted patients had variable RI trends while nonreadmitted patients had uniformly positive interval differences. ${ }^{[8]}$

For comparison, length of stay was empirically chosen as another potential predictor of readmission; this model was not significant. In addition, neither the RI score at discharge nor the interval difference in RI from immediately postoperative to discharge appeared to predict readmission latency. Further, analysis of time to readmission was limited by skew and lack of variance in the data. Intuitively, one might expect a positive correlation between $\mathrm{RI}_{d}$ and the number of days to readmission. However, more than half of the patients readmitted within seven days had $\mathrm{RI}_{d}>70$. Therefore, there may be certain subpopulations of patients or specific RI input variables that result in a high $\mathrm{RI}_{d}$, but are associated with early readmission. $\mathrm{RI}_{d}$ may also have greater efficacy in predicting specific readmission diagnoses such as bleeding and infection; specific components of RI may be more sensitive to clinical data associated with these complications. Although a higher RI threshold for discharge could be chosen, this would also result in some patients staying longer than necessary. Further study is needed to evaluate RI predictors of readmission and latency.

The strengths of our study include the use of a single center, as well as the performance of all surgical procedures by a 
single gynecologic surgeon with over 20 years of experience. Therefore, there was likely a consistency in surgical and postoperative care and clinical management decisions. Also, the RI was not accessed by the gynecologic oncology service during the study interval, which reduced bias. The use of a single center allows for a consistent group of nursing personnel performing all of the nurse's assessments for both cases and controls. The use of a single laboratory center allows for laboratory quality measures to be consistent between groups. In addition, we matched cases and controls for known risk factors for infection and other morbidities.

Limitations of the study include its retrospective design and a small sample size. However, the data placed into the RI system were entered in real time, and the parameters included in the index never changed. To obtain a larger sample, data from a multi-center population or a large gynecologic oncology group would need to be studied. The increase in statistical power gained by these adjustments would have to be weighed against the potential increase in the heterogeneity in patient care and assessment.
In conclusion, the RI value on the day of discharge is helpful in identifying postoperative gynecologic oncology service patients at increased risk for a 30-day readmission. This finding is consistent with previous literature in the medical-surgical population. Because the RI is automatically computed, readily available in the EMR, and quantitative, it is a potentially useful tool for decision-making and for communicating patient status within the gynecologic oncology healthcare team. Delaying discharge for patients with RI below a designated threshold may reduce readmission rates in postoperative gynecologic oncology patients. An important area for further research would be the cost-effectiveness of explicitly incorporating the RI into the EMR for healthcare decisions.

\section{ACKNOWLEDGEMENTS}

The authors thank Larry Leemis $\mathrm{PhD}$ for statistical analysis support and Eric Dieffenwierth for assistance in completing this work.

\section{CONFLICTS OF INTEREST Disclosure}

The authors declare they have no conflicts of interest.

\section{REFERENCES}

[1] Ward KK, Shah NR, Davis MA, et al. Creating a risk of readmission (ROR) score for gynecologic oncology (GO) patient. JCO. 2013; 31(15_suppl): 6636-6636.

[2] Henretta MS, Scalici JM, Engelhard CL, et al. The revolving door: Hospital readmissions of gynecologic oncology patients. Gynecologic Oncology. 2011; 122(3): 479-483. https ://doi .org/10.1 016/j.ygyno. 2011.05.011

[3] Wilbur MB, Mannschreck DB, Angarita AM, et al. Unplanned 30day hospital readmission as a quality measure in gynecologic oncology. Gynecologic Oncology. 2016; 143: 604-10. PMid:27665313. https://doi.org/10.1016/j.ygyno.2016.09.020

[4] Uppal S, Penn C, del Carmen MG, et al. Readmissions after major gynecologic oncology surgery. Gynecologic Oncology. 2016; 141: 287-92. PMid:26927757. https ://doi .org/10.1016/j . ygyno. 2016.02 .031

[5] Eskander RN, Chang J, Ziogas A, et al. Evaluation of 30-Day Hospital Readmission After Surgery for Advanced-Stage Ovarian Cancer in a Medicare Population. JCO. 2014; 32(36): 4113-4119. PMid:25385738. https://doi.org/10.1200/JC0.2014.56.77 43

[6] Zuckerman RB, Sheingold SH, Orav EJ, et al. Readmissions, Observation, and the Hospital Readmissions Reduction Program. New England Journal of Medicine. 2016; 374(16): 1543-1551. PMid:26910198. https://doi.org/10.1056/NEJMsa1513024
[7] Rothman MJ, Rothman SI, Beals J. Development and validation of a continuous measure of patient condition using the Electronic Medical Record. Journal of Biomedical Informatics. 2013; 46(5): 837-848. PMid:23831554. https://doi.org/10.1016/j.jbi.2013.06. 011

[8] Piper GL, Kaplan LJ, Maung AA, et al. Using the Rothman index to predict early unplanned surgical intensive care unit readmissions. J Trauma Acute Care Surg. 2014; 77(1): 78-82. PMid:24977759. https://doi.org/10.1097/TA.0000000000000265

[9] Bradley E, Yakusheva O, Horwitz LI, et al. Identifying Patients at Increased Risk for Unplanned Readmission. Medical care. 2013; 51(9): 761-766. PMid:23942218. https://doi.org/10.1097/MLR.0b 013e3182a0f 492

[10] Finlay GD, Rothman MJ, Smith RA. Measuring the modified early warning score and the Rothman Index: Advantages of utilizing the electronic medical record in an early warning system. Journal of Hospital Medicine. 2014; 9(2): 116-119. PMid:24357519. https://doi.org/10.1002/jhm.2132

[11] da Silva YS, Hamilton MF, Horvat C, et al. Evaluation of Electronic Medical Record Vital Sign Data Versus a Commercially Available Acuity Score in Predicting Need for Critical Intervention at a Tertiary Children's Hospital. Pediatr Crit Care Med. 2015; 16(7): 644-651. PMid:25901545. https://doi.org/10.1097/PCC. 0000000000 000444 